\title{
Dites non au statu quo!
}

\author{
par Glen Brown
}

$\mathrm{L}$ orsque vous prodiguez des soins pharmaceutiques à vos patients, vous intervenez pour modifier la pharmacothérapie de chaque patient afin de lui donner les meilleures chances d'atteindre les objectifs thérapeutiques souhaités. Cela représente un grand service pour vos patients, et des milliers de Canadiens de tout le pays bénéficient de la participation des pharmaciens à leurs soins de santé. Cependant, plusieurs des problèmes qui nécessitent l'intervention des pharmaciens sont récurrents. Par exemple, vous êtes probablement intervenu de nombreuses fois pour modifier la dose d'un médicament à cause de la clairance d'un médicament qui était insuffisante ou qui s'était améliorée, ce qui arrive avec les changements de la fonction rénale. De même, un intérêt récent pour le bilan comparatif des médicaments révèle que des interventions pour corriger les pharmacothérapies inappropriées ont été souvent nécessaires aux points de transfert des soins. Le défi pour tous les fournisseurs de soins, dont les pharmaciens, est de reconnaître les situations où la pharmacothérapie est sans cesse sous-optimale et d'amorcer les démarches nécessaires pour que les prochains patients n'aient pas les mêmes problèmes. Fort de ces connaissances, reconnaissez-vous les facteurs qui contribuent aux soins sous-optimaux et prenez-vous des mesures pour corriger ces problèmes?

Dans ce numéro du JCPH, Louie et ses collaborateurs ${ }^{1}$ décrivent un milieu de pratique particulier dans les hôpitaux canadiens où, suggèrent-t-ils, les pharmaciens et les autres fournisseurs de soins ne sont pas adéquatement organisés pour consigner systématiquement les erreurs de médication, en évaluer les causes et prévenir les problèmes pharmacothérapeutiques ultérieurs. Ces chercheurs ont choisi d'examiner les unités de soins intensifs à cause de la nature critique de l'état des patients, du rôle important de la pharmacothérapie dans l'évolution de l'état de santé et du potentiel de conséquences délétères des erreurs de médication. Or, leurs résultats pourraient être extrapolés à de nombreux autres milieux où les pharmaciens contribuent de façon considérable aux soins. Louie et ses collaborateurs cherchaient des méthodes structurées pour signaler, évaluer et réagir aux erreurs de médication, mais je suis certain que leur analyse pourrait être élargie pour englober tous les types de problèmes reliés à la pharmacothérapie. Je ne vous dis pas que chaque intervention du pharmacien est en réponse à une erreur de médication, mais je crois que le questionnement des auteurs sur la raison pour laquelle les interventions des pharmaciens ne sont pas utilisées comme méthode de dépistage des soins sous-optimaux est valide. Combien de fois vous êtes-vous arrêté pour vous poser cette question: "Pourquoi dois-je réaliser cette intervention? ", plutôt que de simplement aller de l'avant avec l'intervention? Je soupçonne que vous intervenez tout simplement beaucoup plus souvent que vous n'examinez les causes des problèmes reliés à la pharmacothérapie récurrents. En agissant ainsi, en tant que pharmaciens, ne favorisons-nous pas la récurrence de ces problèmes? N'acceptons-nous pas trop facilement le statu quo comme le cours inévitable des événements?

Le défi pour nous tous, quel que soit notre milieu de praitque, est de voir au-delà de chaque patient et de ses problèmes et de rechercher les causes ou les habitudes potentiellement corrigibles qui pourraient être à l'origine du problème. Un élément clé de cette démarche est le recours à un système permettant de consigner les problèmes reliés à la pharmacothérapie et les facteurs ayant pu y contribuer. Louie et ses collaborateurs ont utilisé les erreurs de médication comme un exemple de problème relié à la pharmacothérapie intéressant à surveiller et à examiner, mais d'autres exemples, comme la sélection des médicaments, la durée du traitement ou la posologie du médicament pourraient aussi être valables. De nombreuses pharmacies hospitalières au Canada tiennent un système de mesure de la charge de travail des pharmaciens où sont consignés les types d'interventions. Ce serait un petit pas en avant que d'inclure les données sur les processus entourant les médicaments et les maladies associés à l'intervention ${ }^{2}$. Louie et ses collaborateurs suggèrent que de nombreux pharmaciens au Canada pratiquent dans un environnement où les déclarations volontaires manuscrites sont la norme. Ils font remarquer à juste titre que la déclaration volontaire est inadéquate pour attester la fréquence des erreurs de médication et les facteurs y ayant 
contribué. D’autres ont découvert que les déclarations électroniques sont plus efficaces et plus complètes pour permettre de dépister les problèmes pharmacothérapeutiques ${ }^{3}$. La consignation adéquate permettra de cerner les habitudes d'utilisation des médicaments ou les combinaisons médicament-maladie qui sont fréquemment associées à des problèmes reliés à la pharmacothérapie commandant une intervention. Un examen plus poussé des facteurs ayant contribué à ces problèmes devrait aider à définir les étapes qui pourraient être modifiées pour minimiser la récurrence des problèmes reliés à la pharmacothérapie.

Les pharmaciens doivent jouer un rôle actif dans l'élaboration de processus permettant d'étudier systématiquement la source des problèmes reliés à la pharmacothérapie ${ }^{3,4}$. Dites non au statu quo, et soyez des agents de changement! Vos futurs patients en bénéficieront. Le Journal fait bon accueil aux rapports sur la façon dont vous avez mis de l'avant des processus pour consigner et évaluer de manière systématique un aspect particulier ou tous les aspects des problèmes pharmacothérapeutiques pour que les autres puissent apprendre de vos réussites.

[Traduction par l'éditeur]
Références

1. Louie K, Wilmer A, Wong H, Grubisic M, Ayas N, Dodek P. Medication error reporting systems: a survey of Canadian intensive care units. Can J Hosp Pharm 2010;63(1):20-24.

2. Raybardhan S, Balen RM, Partovi N, Loewen P, Liu G, Jewesson PJ. Documenting drug-related problems with personal digital assistants in a multisite health system. Am J Health Syst Pharm 2005;62(17):1782-1787.

3. Savage SW, Schneider PJ, Pedersen CA. Utility of an online medicationerror-reporting system. Am J Health Syst Pharm 2005;62(21):2265-2270.

4. Costello JL, Torowicz DL, Yeh TS. Effects of a pharmacist-led pediatrics medication safety team on medication-error reporting. Am J Health Syst Pharm 2007;64(13):1422-1426.

Glen Brown, B. Sc. Pharm., Pharm. D, FCSHP, BCPS, est spécialiste en pharmacie clinique, Service de pharmacie, St Paul's Hospital de Vancouver, en Colombie-Britannique. II est également rédacteur adjoint du $J C P H$.

Adresse de correspondance :

D' Glen Brown

Pharmacy Department

St Paul's Hospital

1081 Burrard Street

Vancouver (C.-B.) V6H 1 G7

Courriel : gbrown@providencehealth.bc.ca

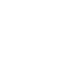

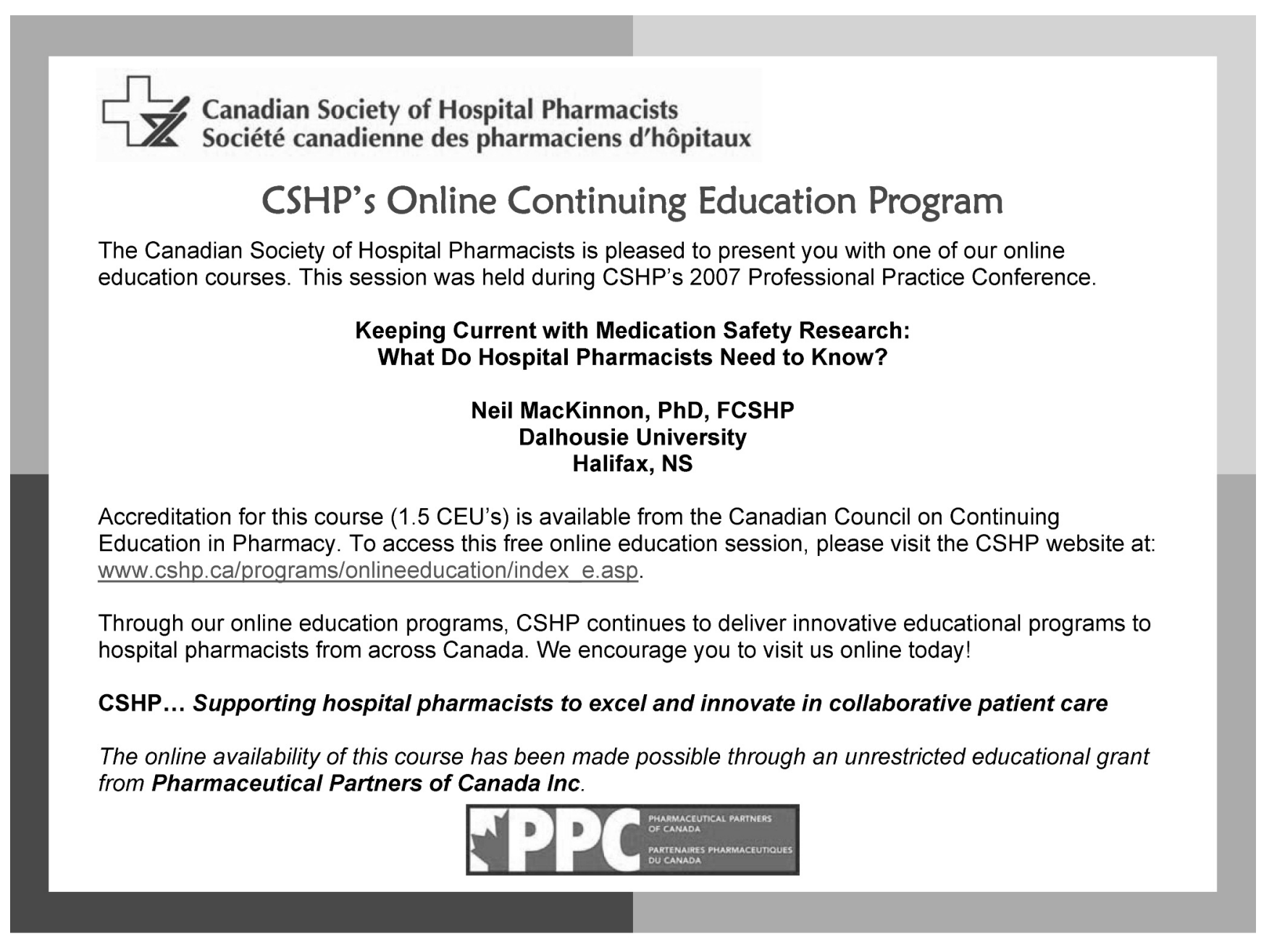

